\title{
Order Parameters, Broken Symmetry, and Topology
}

\author{
James P. Sethna \\ Laboratory of Applied Physics, Technical University of Denmark, \\ DK-2800 Lyngby, DENMARK, and NORDITA, DK-2100 Copenhagen Ø, \\ DENMARK and Laboratory of Atomic and Solid State Physics (LASSP), \\ Clark Hall, Cornell University, Ithaca, NY 14853-2501, USA
}

(Dated: June 16, 2009, 1:00 am)

\begin{abstract}
We introduce the theoretical framework we use to study the bewildering variety of phases in condensed-matter physics. We emphasize the importance of the breaking of symmetries, and develop the idea of an order parameter through several examples. We discuss elementary excitations and the topological theory of defects.

1991 Lectures in Complex Systems, Eds. L. Nagel and D. Stein, Santa Fe Institute Studies in the Sciences of Complexity, Proc. Vol. XV, Addison-Wesley, 1992.
\end{abstract}

PACS numbers:

Keywords:

As a kid in elementary school, I was taught that there were three states of matter: solid, liquid, and gas. The ancients thought that there were four: earth, water, air, and fire, which was considered sheer superstition. In junior high, I remember reading a book called The Seven States of Matter. At least one was "plasma", which made up stars and thus most of the universe, [4] and which sounded rather like fire to me.
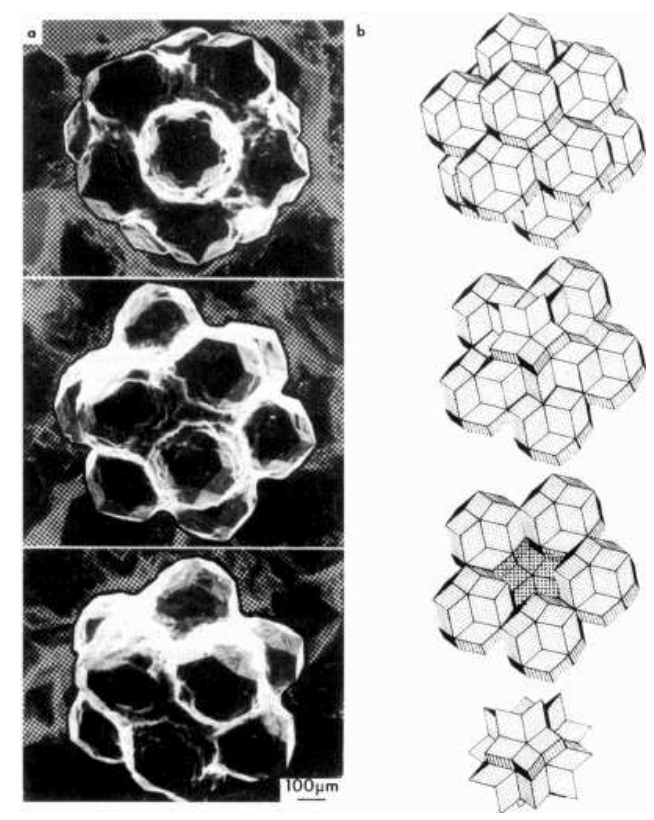

FIG. 1: Quasicrystals. Much of these two lectures will discuss the properties of crystals. Crystals are surely the oldest known of the broken-symmetry phases of matter, and remain the most beautiful illustrations. It's amazing that in the past few years, we've uncovered an entirely new class of crystals. Shown here is a photograph of a quasicrystalline metallic alloy, with icosahedral symmetry. Notice that the facets are pentagonal: our old notions of crystals had to be completely revised to include this type of symmetry.

The original three, by now, have become multitudes.
In important and precise ways, magnets are a distinct form of matter. Metals are different from insulators. Superconductors and superfluids are striking new states of matter. The liquid crystal in your wristwatch is one of a huge family of different liquid crystalline states of matter 2] (nematic, cholesteric, blue phase I, II, and blue fog, smectic A, B, C, C* $, \mathrm{D}, \mathrm{I}, \ldots)$. There are over 200 qualitatively different types of crystals, not to mention the quasicrystals (figure 1). There are disordered states of matter like spin glasses, and states like the fractional quantum hall effect with excitations of charge $e / 3$ like quarks. Particle physicists tell us that the vacuum we live within has in the past been in quite different states: in the last vacuum but one, there were four different kinds of light [3] (mediated by what is now the photon, the $\mathrm{W}^{+}$, the $\mathrm{W}^{-}$, and the $\mathrm{Z}$ particle). We'll discuss this more in lecture two.

When there were only three states of matter, we could learn about each one and then turn back to learning long division. Now that there are multitudes, though, we've had to develop a system. Our system is constantly being extended and modified, because we keep finding new phases which don't fit into the old frameworks. It's amazing how the 500th new state of matter somehow screws up a system which worked fine for the first 499. Quasicrystals, the fractional quantum hall effect, and spin glasses all really stretched our minds until (1) we understood why they behaved the way they did, and (2) we understood how they fit into the general framework.

In this lecture, I'm going to tell you the system. In the next three lectures, I'll discuss some gaps in the system: materials and types of behavior which don't fit into the neat framework presented here. I'll try to maximize the number of pictures and minimize the number of formulas, but (particularly in lecture III) there are problems and ideas that I don't understand well enough to explain simply. Most of what I tell you in this lecture is both true and important. Much of what is contained in the next 
three lectures represents my own pet ideas and theories, and you should be warned not to take my messages there as gospel.

The system consists of four basic steps. 1] First, you must identify the broken symmetry. Second, you must define an order parameter. Third, you are told to examine the elementary excitations. Fourth, you classify the topological defects. Most of what I say I take from Mermin [1], Coleman [3], and deGennes [2], and I heartily recommend these excellent articles to my audience. We take each step in turn.

\section{IDENTIFY THE BROKEN SYMMETRY}

What is it which distinguishes the hundreds of different states of matter? Why do we say that water and olive oil are in the same state (the liquid phase), while we say aluminum and (magnetized) iron are in different states? Through long experience, we've discovered that most phases differ in their symmetry. [5]

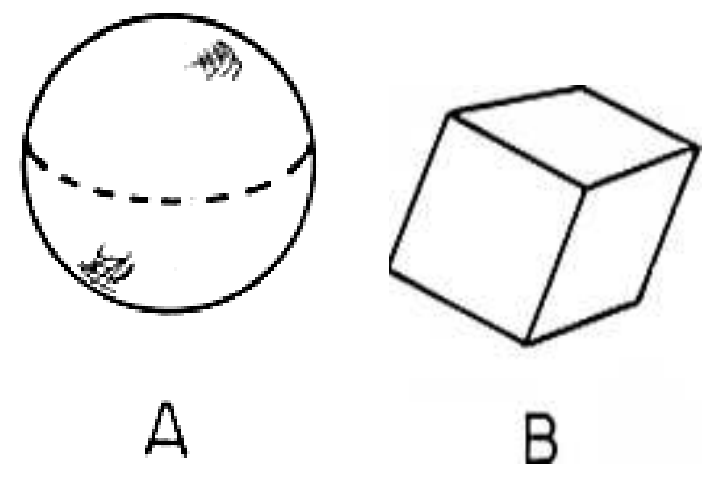

FIG. 2: Which is more symmetric? The cube has many symmetries. It can be rotated by $90^{\circ}, 180^{\circ}$, or $270^{\circ}$ about any of the three axes passing through the faces. It can be rotated by $120^{\circ}$ or $240^{\circ}$ about the corners, and by $180^{\circ}$ about an axis passing from the center through any of the 12 edges. The sphere, though, can be rotated by any angle. The sphere respects rotational invariance: all directions are equal. The cube is an object which breaks rotational symmetry: once the cube is there, some directions are more equal than others.

Consider figure 2, showing a cube and a sphere. Which is more symmetric? Clearly, the sphere has many more symmetries than the cube. One can rotate the cube by $90^{\circ}$ in various directions and not change its appearance, but one can rotate the sphere by any angle and keep it unchanged.

In figure 3 , we see a 2 -D schematic representation of ice and water. Which state is more symmetric here? Naively, the ice looks much more symmetric: regular arrangements of atoms forming a lattice structure. The water looks irregular and disorganized. On the other hand, if one rotated figure $3 \mathrm{~B}$ by an arbitrary angle, it would still look like water! Ice has broken rotational symmetry: one

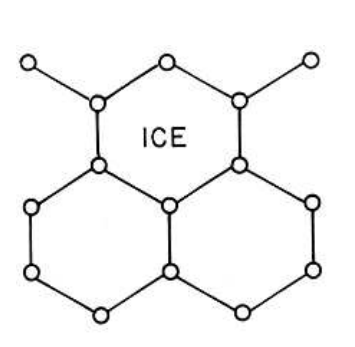

A

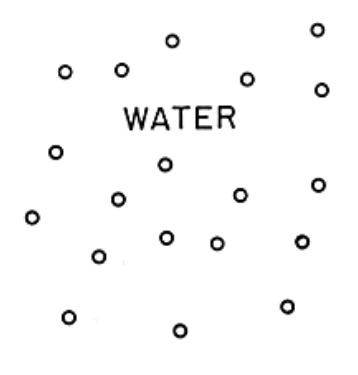

B
FIG. 3: Which is more symmetric? At first glance, water seems to have much less symmetry than ice. The picture of "two-dimensional" ice clearly breaks the rotational invariance: it can be rotated only by $120^{\circ}$ or $240^{\circ}$. It also breaks the translational invariance: the crystal can only be shifted by certain special distances (whole number of lattice units). The picture of water has no symmetry at all: the atoms are jumbled together with no long-range pattern at all. Water, though, isn't a snapshot: it would be better to think of it as a combination of all possible snapshots! Water has a complete rotational and translational symmetry: the pictures will look the same if the container is tipped or shoved.

can rotate figure $3 \mathrm{~A}$ only by multiples of $60^{\circ}$. It also has a broken translational symmetry: it's easy to tell if the picture is shifted sideways, unless one shifts by a whole number of lattice units. While the snapshot of the water shown in the figure has no symmetries, water as a phase has complete rotational and translational symmetry.

One of the standard tricks to see if two materials differ by a symmetry is to try to change one into the other smoothly. Oil and water won't mix, but I think oil and alcohol do, and alcohol and water certainly do. By slowly adding more alcohol to oil, and then more water to the alcohol, one can smoothly interpolate between the two phases. If they had different symmetries, there must be a first point when mixing them when the symmetry changes, and it is usually easy to tell when that phase transition happens.

\section{DEFINE THE ORDER PARAMETER}

Particle physics and condensed-matter physics have quite different philosophies. Particle physicists are constantly looking for the building blocks. Once pions and protons were discovered to be made of quarks, they became demoted into engineering problems. Now that quarks and electrons and photons are made of strings, and strings are hard to study (at least experimentally), there is great anguish in the high-energy community. Condensed-matter physicists, on the other hand, try to understand why messy combinations of zillions of electrons and nuclei do such interesting simple things. To them, the fundamental question is not discovering the 
underlying quantum mechanical laws, but in understanding and explaining the new laws that emerge when many particles interact.

As one might guess, we don't keep track of all the electrons and protons. [6] We're always looking for the important variables, the important degrees of freedom. In a crystal, the important variables are the motions of the atoms away from their lattice positions. In a magnet, the important variable is the local direction of the magnetization (an arrow pointing to the "north" end of the local magnet). The local magnetization comes from complicated interactions between the electrons, and is partly due to the little magnets attached to each electron and partly due to the way the electrons dance around in the material: these details are for many purposes unimportant.

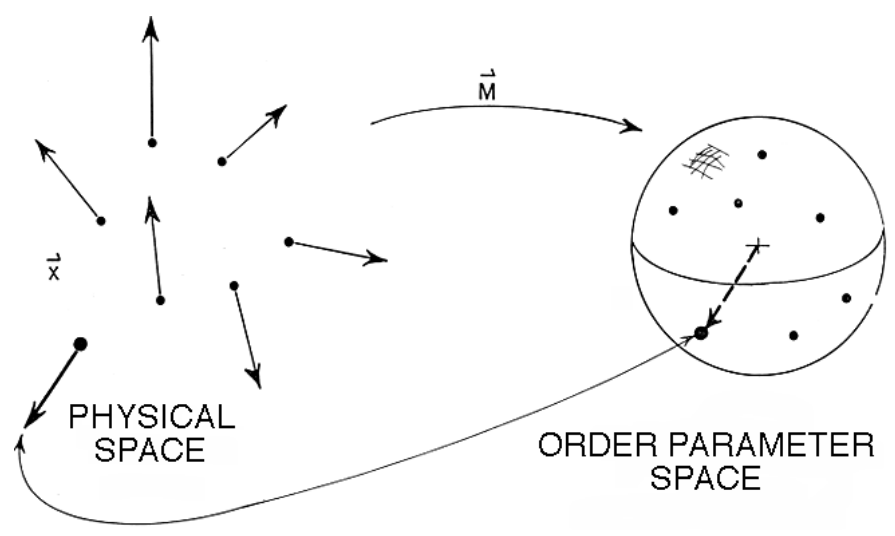

FIG. 4: Magnet. We take the magnetization $\vec{M}$ as the order parameter for a magnet. For a given material at a given temperature, the amount of magnetization $|\vec{M}|=M_{0}$ will be pretty well fixed, but the energy is often pretty much independent of the direction $\hat{M}=\vec{M} / M_{0}$ of the magnetization. (You can think of this as a arrow pointing to the north end of each atomic magnet.) Often, the magnetization changes directions smoothly in different parts of the material. (That's why not all pieces of iron are magnetic!) We describe the current state of the material by an order parameter field $\vec{M}(\mathbf{x})$.

The order parameter field is usually thought of as an arrow at each point in space. It can also be thought of as a function taking points in space $\mathbf{x}$ into points on the sphere $|\vec{M}|=M_{0}$. This sphere $\mathcal{S}^{2}$ is the order parameter space for the magnet.

The important variables are combined into an "order parameter field".7] In figure 4, we see the order parameter field for a magnet. [8] At each position $\mathbf{x}=(x, y, z)$ we have a direction for the local magnetization $\vec{M}(\mathbf{x})$. The length of $\vec{M}$ is pretty much fixed by the material, but the direction of the magnetization is undetermined. By becoming a magnet, this material has broken the rotational symmetry. The order parameter $\vec{M}$ labels which of the various broken symmetry directions the material has chosen.

The order parameter is a field: at each point in our magnet, $\vec{M}(\mathbf{x})$ tells the local direction of the field near x. Why do we do this? Why would the magnetization point in different directions in different parts of the magnet? Usually, the material has lowest energy when the order parameter field is uniform, when the symmetry is broken in the same way throughout space. In practise, though, the material often doesn't break symmetry uniformly. Most pieces of iron don't appear magnetic, simply because the local magnetization points in different directions at different places. The magnetization is already there at the atomic level: to make a magnet, you pound the different domains until they line up. We'll see in this lecture that most of the interesting behavior we can study involves the way the order parameter varies in space.

The order parameter field $\vec{M}(\mathbf{x})$ can be usefully visualized in two different ways. On the one hand, one can think of a little vector attached to each point in space. On the other hand, we can think of it as a mapping from real space into order parameter space. That is, $\vec{M}$ is a function which takes different points in the magnet onto the surface of a sphere (figure 4). Mathematicians call the sphere $\mathrm{S}^{2}$, because it locally has two dimensions. (They don't care what dimension the sphere is embedded in.)

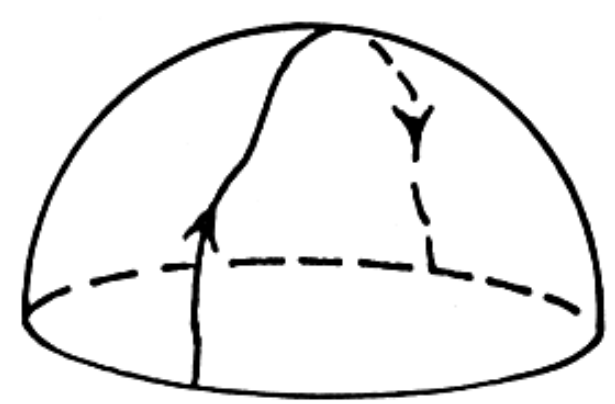

FIG. 5: Nematic liquid crystal. Nematic liquid crystals are made up of long, thin molecules that prefer to align with one another. (Liquid crystal watches are made of nematics.) Since they don't care much which end is up, their order parameter isn't precisely the vector $\hat{n}$ along the axis of the molecules. Rather, it is a unit vector up to the equivalence $\hat{n} \equiv-\hat{n}$. The order parameter space is a half-sphere, with antipodal points on the equator identified. Thus, for example, the path shown over the top of the hemisphere is a closed loop: the two intersections with the equator correspond to the same orientations of the nematic molecules in space.

Before varying our order parameter in space, let's develop a few more examples. The liquid crystal in LCD displays (like those in digital watches) are nematics. Nematics are made of long, thin molecules which tend to line up so that their long axes are parallel. Nematic liquid crystals, like magnets, break the rotational symmetry. Unlike magnets, though, the main interaction isn't to line up the north poles, but to line up the axes. (Think of the molecules as American footballs: the same up and down.) Thus the order parameter isn't a vector $\vec{M}$ but 
a headless vector $\vec{n} \equiv-\vec{n}$. The order parameter space is a hemisphere, with opposing points along the equator identified (figure 5). This space is called $\mathcal{R} \mathcal{P}^{2}$ by the mathematicians (the projective plane), for obscure reasons.

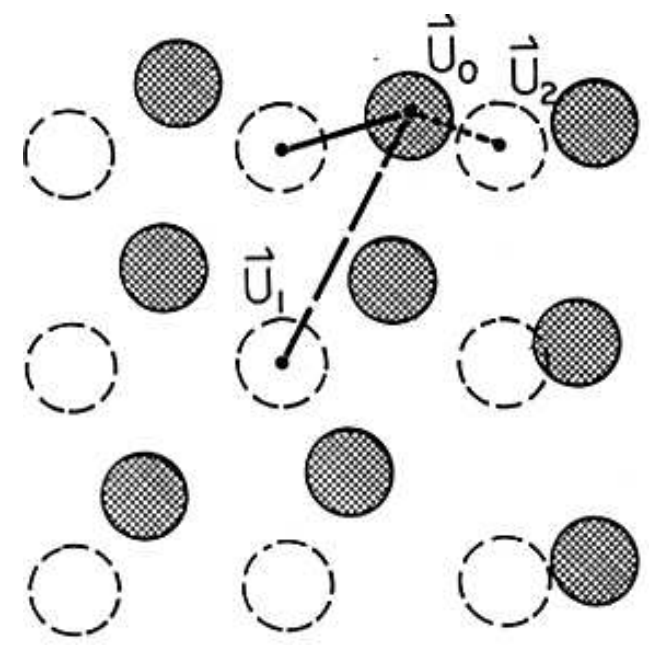

FIG. 6: Two dimensional crystal. A crystal consists atoms arranged in regular, repeating rows and columns. At high temperatures, or when the crystal is deformed or defective, the atoms will be displaced from their lattice positions. The displacements $\vec{u}$ are shown. Even better, one can think of $u(\mathbf{x})$ as the local translation needed to bring the ideal lattice into registry with atoms in the local neighborhood of $\mathbf{x}$.

Also shown is the ambiguity in the definition of $u$. Which "ideal" atom should we identify with a given "real" one? This ambiguity makes the order parameter $u$ equivalent to $u+\operatorname{ma} \hat{x}+n a \hat{y}$. Instead of a vector in two dimensional space, the order parameter space is a square with periodic boundary conditions.

For a crystal, the important degrees of freedom are associated with the broken translational order. Consider a two-dimensional crystal which has lowest energy when in a square lattice, but which is deformed away from that configuration (figure 6). This deformation is described by an arrow connecting the undeformed ideal lattice points with the actual positions of the atoms. If we are a bit more careful, we say that $\vec{u}(\mathbf{x})$ is that displacement needed to align the ideal lattice in the local region onto the real one. By saying it this way, $\vec{u}$ is also defined between the lattice positions: there still is a best displacement which locally lines up the two lattices.

The order parameter $\vec{u}$ isn't really a vector: there is a subtlety. In general, which ideal atom you associate with a given real one is ambiguous. As shown in figure 6 , the displacement vector $\vec{u}$ changes by a multiple of the lattice constant $a$ when we choose a different reference atom:

$$
\vec{u} \equiv \vec{u}+a \hat{x}=\vec{u}+m a \hat{x}+n a \hat{y} .
$$

The set of distinct order parameters forms a square with periodic boundary conditions. As figure 7 shows, a
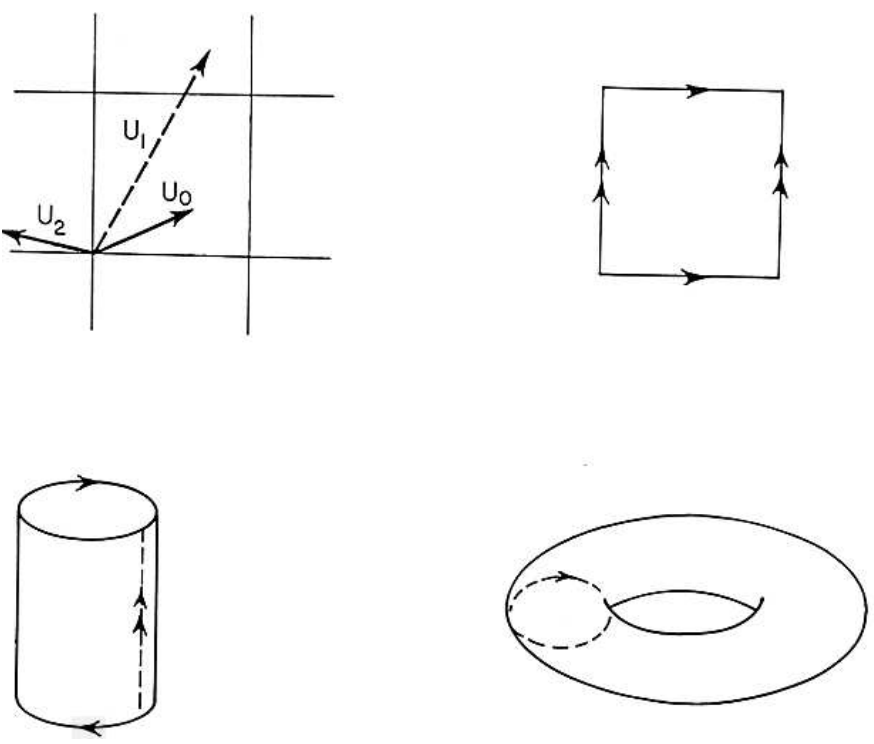

FIG. 7: Order parameter space for a two-dimensional crystal. Here we see that a square with periodic boundary conditions is a torus. (A torus is a surface of a doughnut, inner tube, or bagel, depending on your background.)

square with periodic boundary conditions has the same topology as a torus, $\mathrm{T}^{2}$. (The torus is the surface of a doughnut, bagel, or inner tube.)

Finally, let's mention that guessing the order parameter (or the broken symmetry) isn't always so straightforward. For example, it took many years before anyone figured out that the order parameter for superconductors and superfluid Helium 4 is a complex number $\psi$. The order parameter field $\psi(\mathbf{x})$ represents the "condensate wave function", which (extremely loosely) is a single quantum state occupied by a large fraction of the Cooper pairs or helium atoms in the material. The corresponding broken symmetry is closely related to the number of particles. In "symmetric", normal liquid helium, the local number of atoms is conserved: in superfluid helium, the local number of atoms becomes indeterminate! (This is because many of the atoms are condensed into that delocalized wave function.) Anyhow, the magnitude of the complex number $\psi$ is a fixed function of temperature, so the order parameter space is the set of complex numbers of magnitude $|\psi|$. Thus the order parameter space for superconductors and superfluids is a circle $\mathrm{S}^{1}$.

Now we examine small deformations away from a uniform order parameter field.

\section{EXAMINE THE ELEMENTARY EXCITATIONS}

Its amazing how slow human beings are. The atoms inside your eyelash collide with one another a million million times during each time you blink your eye. It's 
not surprising, then, that we spend most of our time in condensed-matter physics studying those things in materials that happen slowly. Typically only vast conspiracies of immense numbers of atoms can produce the slow behavior that humans can perceive.

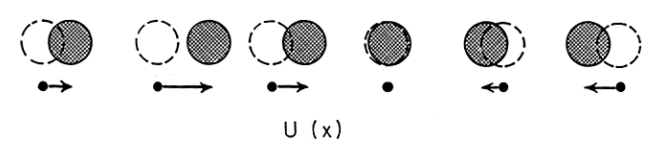

FIG. 8: One dimensional crystal: phonons. The order parameter field for a one-dimensional crystal is the local displacement $u(x)$. Long-wavelength waves in $u(x)$ have low frequencies, and cause sound.

Crystals are rigid because of the broken translational symmetry. Because they are rigid, they fight displacements. Because there is an underlying translational symmetry, a uniform displacement costs no energy. A nearly uniform displacement, thus, will cost little energy, and thus will have a low frequency. These low-frequency elementary excitations are the sound waves in crystals.

A good example is given by sound waves. We won't talk about sound waves in air: air doesn't have any broken symmetries, so it doesn't belong in this lecture. 9] Consider instead sound in the one-dimensional crystal shown in figure 8 . We describe the material with an order parameter field $u(x)$, where here $x$ is the position within the material and $x-u(x)$ is the position of the reference atom within the ideal crystal.

Now, there must be an energy cost for deforming the ideal crystal. There won't be any cost, though, for a uniform translation: $u(x) \equiv u_{0}$ has the same energy as the ideal crystal. (Shoving all the atoms to the right doesn't cost any energy.) So, the energy will depend only on derivatives of the function $u(x)$. The simplest energy that one can write looks like

$$
\mathcal{E}=\int d x(\kappa / 2)(d u / d x)^{2} .
$$

(Higher derivatives won't be important for the low frequencies that humans can hear.) Now, you may remember Newton's law $F=m a$. The force here is given by the derivative of the energy $F=-(d \mathcal{E} / d u)$. The mass is represented by the density of the material $\rho$. Working out the math (a variational derivative and an integration by parts, for those who are interested) gives us the equation

$$
\rho \ddot{u}=\kappa\left(d^{2} u / d x^{2}\right) .
$$

The solutions to this equation

$$
u(x, t)=u_{0} \cos \left(2 \pi\left(x / \lambda-\nu_{\lambda} t\right)\right)
$$

represent phonons or sound waves. The wavelength of the sound waves is $\lambda$, and the frequency is $\nu_{\lambda}$. Plugging 4 into 3 gives us the relation

$$
\nu_{\lambda}=\sqrt{\kappa / \rho} / \lambda .
$$

The frequency gets small only when the wavelength gets large. This is the vast conspiracy: only huge sloshings of many atoms can happen slowly. Why does the frequency get small? Well, there is no cost to a uniform translation, which is what 4 looks like for infinite wavelength. Why is there no energy cost for a uniform displacement? Well, there is a translational symmetry: moving all the atoms the same amount doesn't change their interactions. But haven't we broken that symmetry? That is precisely the point.

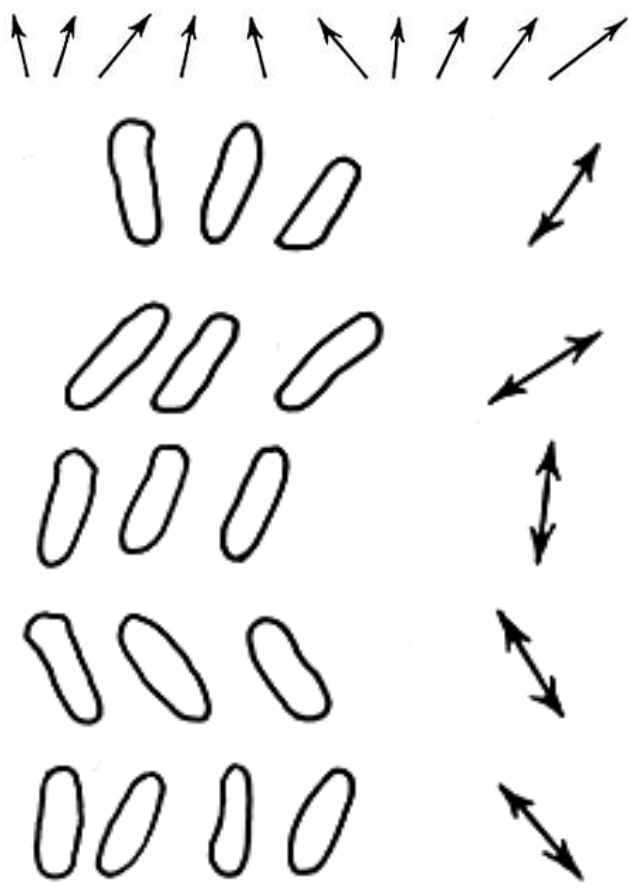

FIG. 9: (a) Magnets: spin waves. Magnets break the rotational invariance of space. Because they resist twisting the magnetization locally, but don't resist a uniform twist, they have low energy spin wave excitations.

(b) Nematic liquid crystals: rotational waves. Nematic liquid crystals also have low-frequency rotational waves.

Long after phonons were understood, Jeffrey Goldstone started to think about broken symmetries and order parameters in the abstract. He found a rather general argument that, whenever a continuous symmetry (rotations, translations, $S U(3), \ldots$ ) is broken, long-wavelength modulations in the symmetry direction should have low frequencies. The fact that the lowest energy state has a broken symmetry means that the system is stiff: modulating the order parameter will cost an energy rather like that in equation 2. In crystals, the broken translational order introduces a rigidity to shear deformations, and low frequency phonons (figure 8). In magnets, the broken rotational symmetry leads to a magnetic stiffness and spin waves (figure 9a). In nematic liquid crystals, the broken rotational symmetry introduces an orientational elastic stiffness (it pours, but resists bending!) 
and rotational waves (figure $9 \mathrm{~b}$ ).

In superfluids, the broken gauge symmetry leads to a stiffness which results in the superfluidity. Superfluidity and superconductivity really aren't any more amazing than the rigidity of solids. Isn't it amazing that chairs are rigid? Push on a few atoms on one side, and $10^{9}$ atoms away atoms will move in lock-step. In the same way, decreasing the flow in a superfluid must involve a cooperative change in a macroscopic number of atoms, and thus never happens spontaneously any more than two parts of the chair ever drift apart.

The low-frequency Goldstone modes in superfluids are heat waves! (Don't be jealous: liquid helium has rather cold heat waves.) This is often called second sound, but is really a periodic modulation of the temperature which passes through the material like sound does through a metal.

O.K., now we're getting the idea. Just to round things out, what about superconductors? They've got a broken gauge symmetry, and have a stiffness to decays in the superconducting current. What is the low energy excitation? It doesn't have one. But what about Goldstone's theorem? Well, you know about physicists and theorems ...

That's actually quite unfair: Goldstone surely had conditions on his theorem which excluded superconductors. Actually, I believe Goldstone was studying superconductors when he came up with his theorem. It's just that everybody forgot the extra conditions, and just remembered that you always got a low frequency mode when you broke a continuous symmetry. We of course understood all along why there isn't a Goldstone mode for superconductors: it's related to the Meissner effect. The high energy physicists forgot, though, and had to rediscover it for themselves. Now we all call the loophole in Goldstone's theorem the Higgs mechanism, because (to be truthful) Higgs and his high-energy friends found a much simpler and more elegant explanation than we had. We'll discuss Meissner effects and the Higgs mechanism in the next lecture.

I'd like to end this section, though, by bringing up another exception to Goldstone's theorem: one we've known about even longer, but which we don't have a nice explanation for. What about the orientational order in crystals? Crystals break both the continuous translational order and the continuous orientational order. The phonons are the Goldstone modes for the translations, but there are no orientational Goldstone modes. [10] We'll discuss this further in the next lecture, but I think this is one of the most interesting unsolved basic questions in the subject.

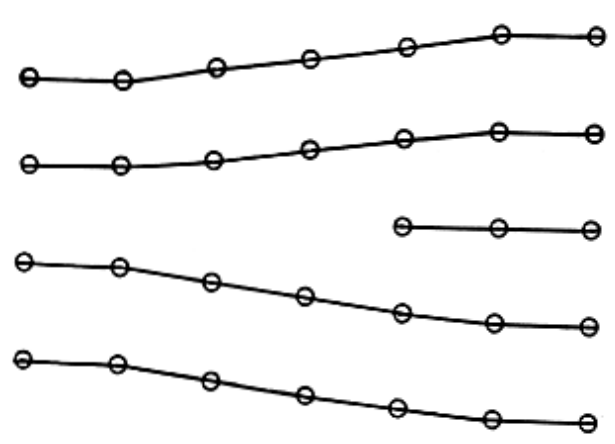

FIG. 10: Dislocation in a crystal. Here is a topological defect in a crystal. We can see that one of the rows of atoms on the right disappears halfway through our sample. The place where it disappears is a defect, because it doesn't locally look like a piece of the perfect crystal. It is a topological defect because it can't be fixed by any local rearrangement. No reshuffling of atoms in the middle of the sample can change the fact that five rows enter from the right, and only four leave from the left!

The Burger's vector of a dislocation is the net number of extra rows and columns, combined into a vector (columns, rows).

\section{CLASSIFY THE TOPOLOGICAL DEFECTS}

When I was in graduate school, the big fashion was topological defects. Everybody was studying homotopy groups, and finding exotic systems to write papers about. It was, in the end, a reasonable thing to do. [11] It is true that in a typical application you'll be able to figure out what the defects are without homotopy theory. You'll spend forever drawing pictures to convince anyone else, though. Most important, homotopy theory helps you to think about defects.

A defect is a tear in the order parameter field. A topological defect is a tear that can't be patched. Consider the piece of 2-D crystal shown in figure 10. Starting in the middle of the region shown, there is an extra row of atoms. (This is called a dislocation.) Away from the middle, the crystal locally looks fine: it's a little distorted, but there is no problem seeing the square grid and defining an order parameter. Can we rearrange the atoms in a small region around the start of the extra row, and patch the defect?

No. The problem is that we can tell there is an extra row without ever coming near to the center. The traditional way of doing this is to traverse a large loop surrounding the defect, and count the net number of rows crossed on the path. In the path shown, there are two rows going up and three going down: no matter how far we stay from the center, there will naturally always be an extra row on the right.

How can we generalize this basic idea to a general problem with a broken symmetry? Remember that the order parameter space for the 2-D square crystal is a torus (see 

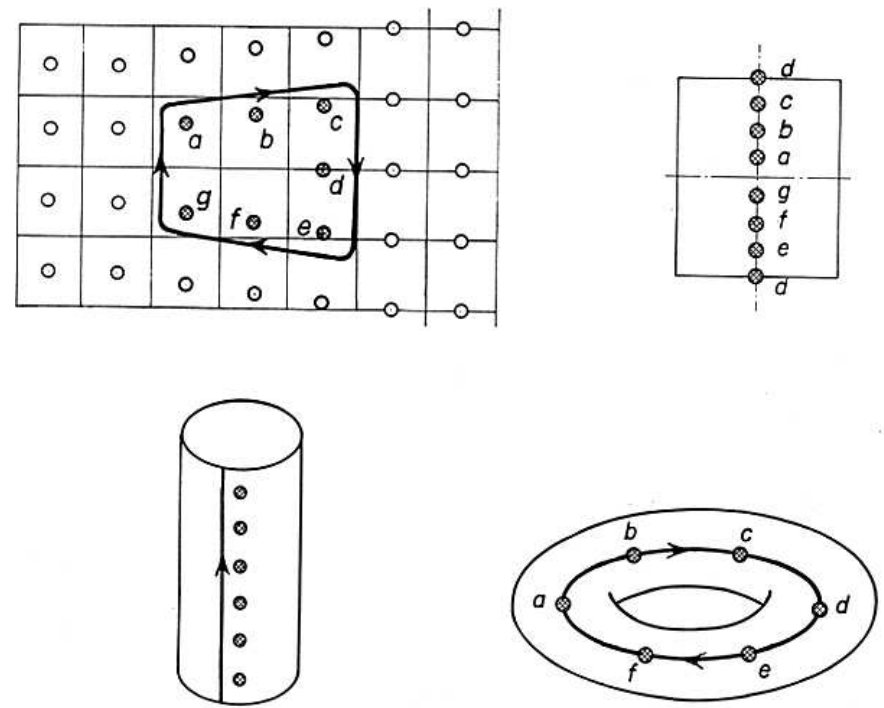

FIG. 11: Loop around the dislocation mapped onto order parameter space. How do we think about our defect in terms of order parameters and order parameter spaces? Consider a closed loop around the defect. The order parameter field $u$ changes as we move around the loop. The positions of the atoms around the loop with respect to their local "ideal" lattice drifts upward continuously as we traverse the loop. This precisely corresponds to a loop around the order parameter space: the loop passes once through the hole in the torus. A loop around the hole corresponds to an extra column of atoms.

Moving the atoms slightly will deform the loop, but won't change the number of times the loop winds through or around the hole. Two loops which traverse the torus the same number of times through and around are equivalent. The equivalence classes are labelled precisely by pairs of integers (just like the Burger's vectors), and the first homotopy group of the torus is $\mathcal{Z} \times \mathcal{Z}$.

figure 7). Remember that the order parameter at a point is that translation which aligns a perfect square grid to the deformed grid at that point. Now, what is the order parameter far to the left of the defect (a), compared to the value far to the right $(\mathrm{d})$ ? Clearly, the lattice to the right is shifted vertically by half a lattice constant: the order parameter has been shifted halfway around the torus. As shown in figure 11, along the top half of a clockwise loop the order parameter (position of the atom within the unit cell) moves upward, and along the bottom half, again moves upward. All in all, the order parameter circles once around the torus. The winding number around the torus is the net number of times the torus is circumnavigated when the defect is orbited once.

This is why they are called topological defects. Topology is the study of curves and surfaces where bending and twisting is ignored. An order parameter field, no matter how contorted, which doesn't wind around the torus can always be smoothly bent and twisted back into a uniform state. If along any loop, though, the order pa- rameter winds either around the hole or through it a net number of times, then enclosed in that loop is a defect which cannot be bent or twisted flat: the winding number can't change by an integer in a smooth and continuous fashion.

How do we categorize the defects for 2-D square crystals? Well, there are two integers: the number of times we go around the central hole, and the number of times we pass through it. In the traditional description, this corresponds precisely to the number of extra rows and columns of atoms we pass by. This was called the Burger's vector in the old days, and nobody needed to learn about tori to understand it. We now call it the first Homotopy group of the torus:

$$
\Pi_{1}\left(\mathcal{T}^{2}\right)=\mathcal{Z} \times \mathcal{Z}
$$

where $\mathcal{Z}$ represents the integers. That is, a defect is labeled by two integers $(m, n)$, where $m$ represents the number of extra rows of atoms on the right-hand part of the loop, and $n$ represents the number of extra columns of atoms on the bottom.

Here's where in the lecture I show the practical importance of topological defects. Unfortunately for you, I can't enclose a soft copper tube for you to play with, the way I do in the lecture. They're a few cents each, and machinists on two continents have been quite happy to cut them up for my demonstrations, but they don't pack well into books. Anyhow, most metals and copper in particular exhibits what is called work hardening. It's easy to bend the tube, but it's amazingly tough to bend it back. The soft original copper is relatively defect-free. To bend, the crystal has to create lots of line dislocations, which move around to produce the bending.[12] The line defects get tangled up, and get in the way of any new defects. So, when you try to bend the tube back, the metal becomes much stiffer. Work hardening has had a noticable impact on the popular culture. The magician effortlessly bends the metal bar, and the strongman can't straighten it ... Superman bends the rod into a pair of handcuffs for the criminals ...

Before we explain why these curves form a group, let's give some more examples of topological defects and how they can be classified. Figure 12a shows a "hedgehog" defect for a magnet. The magnetization simply points straight out from the center in all directions. How can we tell that there is a defect, always staying far away? Since this is a point defect in three dimensions, we have to surround it with a sphere. As we move around on this sphere in ordinary space, the order parameter moves around the order parameter space (which also happens to be a sphere, of radius $|\vec{M}|)$. In fact, the order parameter space is covered exactly once as we surround the defect. This is called the wrapping number, and doesn't change as we wiggle the magnetization in smooth ways. The point defects of magnets are classified by the wrapping 

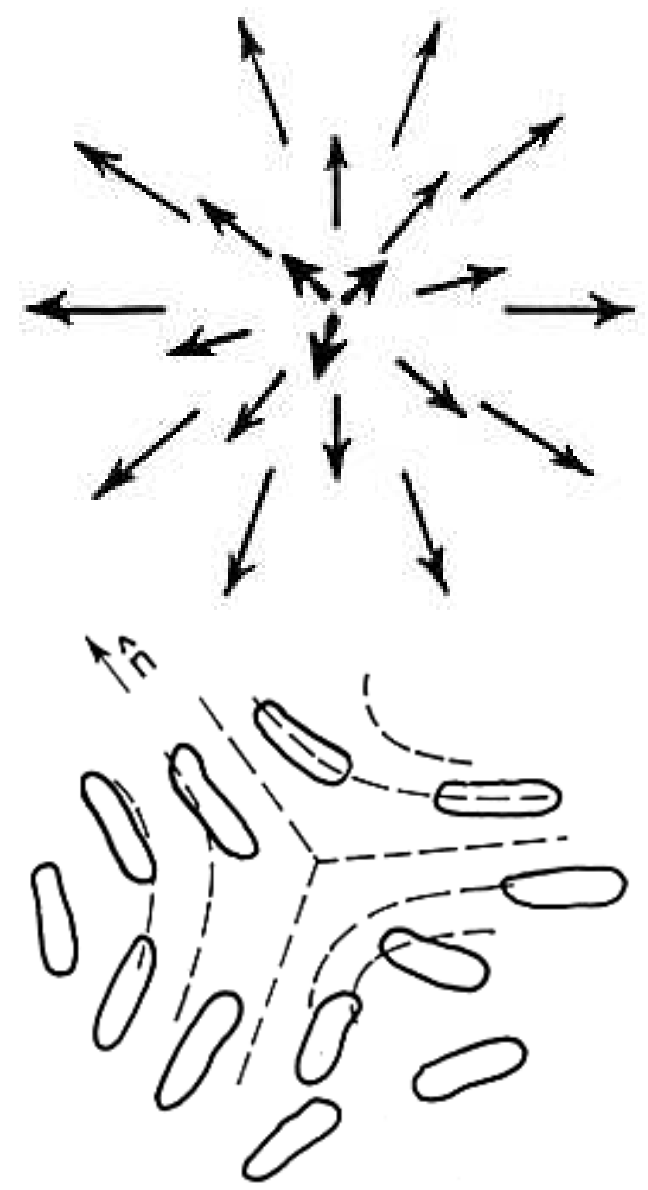

FIG. 12: (a) Hedgehog defect. Magnets have no line defects (you can't lasso a basketball), but do have point defects. Here is shown the hedgehog defect, $\vec{M}(\mathbf{x})=M_{0} \hat{x}$. You can't surround a point defect in three dimensions with a loop, but you can enclose it in a sphere. The order parameter space, remember, is also a sphere. The order parameter field takes the enclosing sphere and maps it onto the order parameter space, wrapping it exactly once. The point defects in magnets are categorized by this wrapping number: the second Homotopy group of the sphere is $\mathcal{Z}$, the integers.

(b) Defect line in a nematic liquid crystal. You can't lasso the sphere, but you can lasso a hemisphere! Here is the defect corresponding to the path shown in figure 5 . As you pass clockwise around the defect line, the order parameter rotates counterclockwise by $180^{\circ}$.

This path on figure 5 would actually have wrapped around the right-hand side of the hemisphere. Wrapping around the left-hand side would have produced a defect which rotated clockwise by $180^{\circ}$. (Imagine that!) The path in figure 5 is halfway in between, and illustrates that these two defects are really not different topologically.

number:

$$
\Pi_{2}\left(\mathcal{S}^{2}\right)=\mathcal{Z}
$$

Here, the 2 subscript says that we're studying the second Homotopy group. It represents the fact that we are surrounding the defect with a $2-\mathrm{D}$ spherical surface, rather than the 1-D curve we used in the crystal. 13.

You might get the impression that a strength 7 defect is really just seven strength 1 defects, stuffed together. You'd be quite right: occasionally, they do bunch up, but usually big ones decompose into small ones. This doesn't mean, though, that adding two defects always gives a bigger one. In nematic liquid crystals, two line defects are as good as none! Magnets didn't have any line defects: a loop in real space never surrounds something it can't smooth out. Formally, the first homotopy group of the sphere is zero: you can't loop a basketball. For a nematic liquid crystal, though, the order parameter space was a hemisphere (figure 5). There is a loop on the hemisphere in figure 5 that you can't get rid of by twisting and stretching. It doesn't look like a loop, but you have to remember that the two opposing points on the equater really represent the same nematic orientation. The corresponding defect has a director field $n$ which rotates $180^{\circ}$ as the defect is orbited: figure $12 \mathrm{~b}$ shows one typical configuration (called an $s=-1 / 2$ defect). Now, if you put two of these defects together, they cancel. (I can't draw the pictures, but consider it a challenging exercise in geometric visualization.) Nematic line defects add modulo 2, like clock arithmetic in elementary school:

$$
\Pi_{1}\left(\mathcal{R} \mathcal{P}^{2}\right)=\mathcal{Z}_{2}
$$

Two parallel defects can coalesce and heal, even though each one individually is stable: each goes halfway around the sphere, and the whole loop can be shrunk to zero.

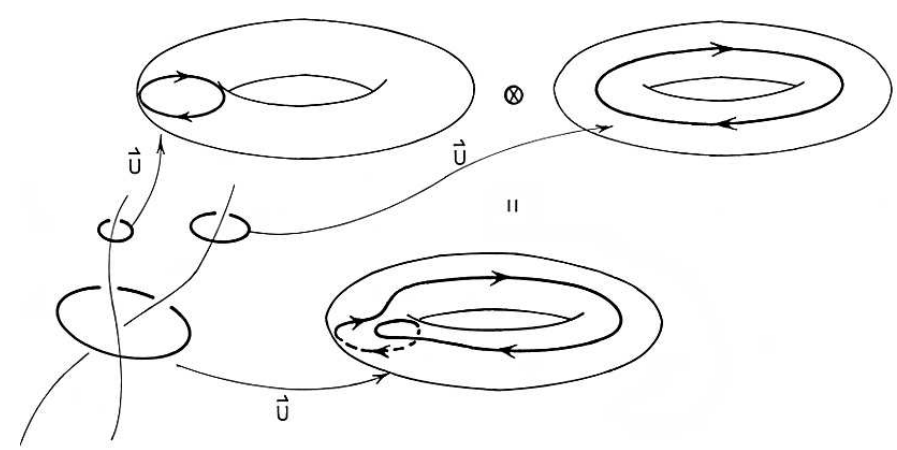

FIG. 13: Multiplying two loops. The product of two loops is given by starting from their intersection, traversing the first loop, and then traversing the second. The inverse of a loop is clearly the same loop travelled backward: compose the two and one can shrink them continuously back to nothing. This definition makes the homotopy classes into a group.

This multiplication law has a physical interpretation. If two defect lines coalesce, their homotopy class must of course be given by the loop enclosing both. This large loop can be deformed into two little loops, so the homotopy class of the coalesced line defect is the product of the homotopy classes of the individual defects.

Finally, why are these defect categories a group? A group is a set with a multiplication law, not necessar- 
ily commutative, and an inverse for each element. For the first homotopy group, the elements of the group are equivalence classes of loops: two loops are equivalent if one can be stretched and twisted onto the other, staying on the manifold at all times. 14] For example, any loop going through the hole from the top (as in the top righthand torus in figure 13) is equivalent to any other one. To multiply a loop $u$ and a loop $v$, one must first make sure that they meet at some point (by dragging them together, probably). Then one defines a new loop $u \otimes v$ by traversing first the loop $u$ and then $v$. 15]

The inverse of a loop $u$ is just the loop which runs along the same path in the reverse direction. The identity element consists of the equivalence class of loops which don't enclose a hole: they can all be contracted smoothly to a point (and thus to one another). Finally, the multiplication law has a direct physical implication: encircling two defect lines of strength $u$ and $v$ is completely equivalent to encircling one defect of strength $u \otimes v$.

This all seems pretty trivial: maybe thinking about order parameter spaces and loops helps one think more clearly, but are there any real uses for talking about the group structure? Let me conclude this lecture with an amazing, physically interesting consequence of the multiplication laws we described. There is a fine discussion of this in Mermin's article [1], but I learned about it from Dan Stein's thesis.

Can two defect lines cross one another? Figure 14a shows two defect lines, of strength (homotopy type) $\alpha$ and $\beta$, which are not parallel. Suppose there is an external force pulling the $\alpha$ defect past the $\beta$ one. Clearly, if we bend and stretch the defect as shown in figure 14b, it can pass by, but there is a trail left behind, of two defect lines. $\alpha$ can really leave $\beta$ behind only if it is topologically possible to erase the trail. Can the two lines annihilate one another? Only if their net strength is zero, as measured by the loop in $14 \mathrm{~b}$.

Now, get two wires and some string. Bend the wires into the shape found in figure $14 \mathrm{~b}$. Tie the string into a fairly large loop, surrounding the doubled portion. Wiggle the string around, and try to get the string out from around the doubled section. You'll find that you can't completely remove the string, (No fair pulling the string past the cut ends of the defect lines!) but that you can slide it downward into the configuration shown in 14c.

Now, in $14 \mathrm{c}$ we see that each wire is encircled once clockwise and once counterclockwise. Don't they cancel? Not necessarily! If you look carefully, the order of traversal is such that the net homotopy class is $\beta \alpha \beta^{-1} \alpha^{-1}$, which is only the identity if $\beta$ and $\alpha$ commute. Thus the physical entanglement problem for defects is directly connected to the group structure of the loops: commutative defects can pass through one another, noncommutative defects entangle.

I'd like to be able to tell you that the work hardening in copper is due to topological entanglements of defects.

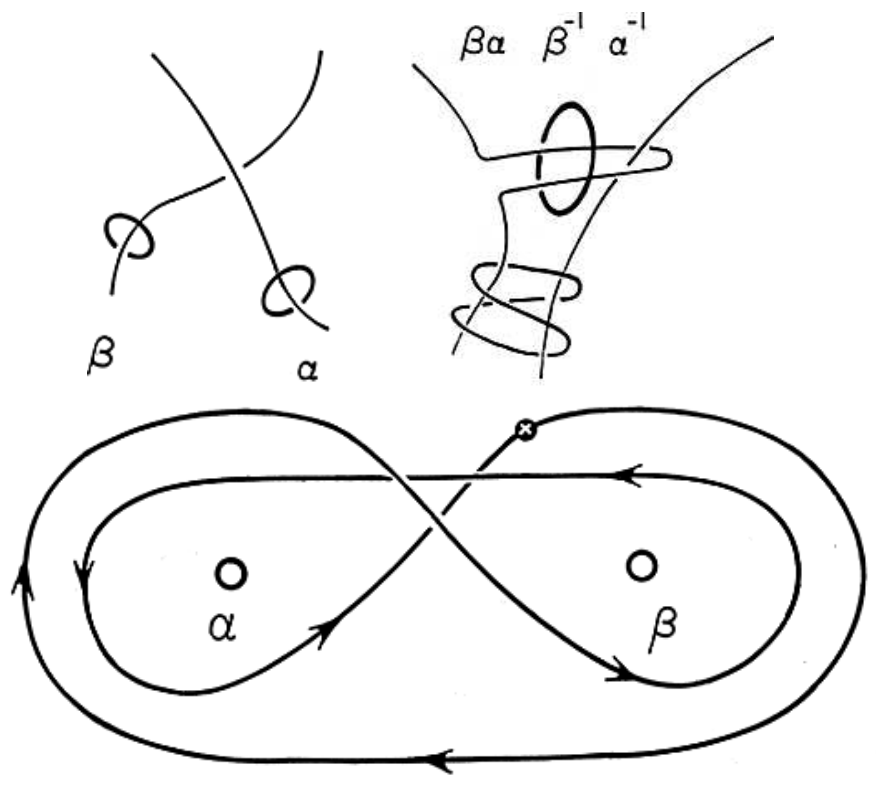

FIG. 14: Defect entanglement. (a) Can a defect line of class $\alpha$ pass by a line of class $\beta$, without getting topologically entangled? (b) We see that we can pass by if we leave a trail: is the connecting double line topologically trivial? Encircle the double line by a loop. The loop can be wiggled and twisted off the double line, but it still circles around the two legs of the defects $\alpha$ and $\beta$. (c) The homotopy class of the loop is precisely $\beta \alpha \beta^{-1} \alpha^{-1}$, which is trivial precisely when $\beta \alpha=$ $\alpha \beta$. Thus two defect lines can pass by one another if their homotopy classes commute!

It wouldn't be true. The homotopy group of dislocation lines in fcc copper is commutative. (It's rather like the 2-D square lattice: if $\alpha=(m, n)$ and $\beta=(o, p)$ with $m, n, o, p$ the number of extra horizontal and vertical lines of atoms, then $\alpha \beta=(m+o, n+p)=\beta \alpha$. $)$ The reason dislocation lines in copper don't pass through one another is energetic, not topological. The two dislocation lines interact strongly with one another, and energetically get stuck when they try to cross. Remember at the beginning of the lecture, I said that there were gaps in the system: the topological theory can only say when things are impossible to do, not when they are difficult to do.

I'd like to be able to tell you that this beautiful connection between the commutativity of the group and the entanglement of defect lines is nonetheless is important in lots of other contexts. That too would not be true. There are two types of materials I know of which are supposed to suffer from defect lines which topological entangle. The first are biaxial nematics, which were thoroughly analyzed theoretically before anyone found one. The other are the metallic glasses, where David Nelson has a theory of defect lines needed to relieve the frustration. We'll discuss closely related theories in lecture 3 . Nelson's defects don't commute, and so can't cross one another. He originally hoped to explain the freezing of 
the metallic glasses into random configurations as an entanglement of defect lines. Nobody has ever been able to take this idea and turn it into a real calculation, though.

Enough, then, of the beautiful and elegant world of homotopy theory: let's begin to think about what order parameter configurations are actually formed in practise.

\section{Acknowledgments}

I'd like to acknowledge NSF grant \# DMR-9118065, and thank NORDITA and the Technical University of Denmark for their hospitality while these lectures were written up.

[1] David Mermin, "The Topological Theory of Defects in Ordered Media", Reviews of Modern Physics 51, 591, 1979.

[2] P. G. de Gennes, The Physics of Liquid Crystals, ch. 1.

[3] Sydney Coleman, "Secret Symmetry: an introduction to spontaneous symmetry breakdown and gauge fields", in Aspects of Symmetry, Selected Erice Lectures, Cambridge University Press, Cambridge, 1985, p. 113, section 1.

[4] They hadn't heard of dark matter back then.

[5] This is not to say that different phases always differ by symmetries! Liquids and gases have the same symmetry. In fact, one can go continuously from a liquid to a gas, by going first to high pressures and then heating. It is safe to say, though, that if the two materials have different symmetries, they are different phases.

[6] The particle physicists use order parameter fields too. Their order parameter fields also hide lots of details about what their quarks and gluons are composed of. The main difference is that they don't know of what their fields are composed. It ought to be reassuring to them that we don't always find our greater knowledge very helpful.

[7] Choosing an order parameter is an art. Usually it's a new phase which we don't understand yet, and guessing the order parameter is a piece of figuring out what's going on. Also, there is often more than one sensible choice. In magnets, for example, one can treat $\vec{M}$ as a fixedlength vector in $\mathcal{S}^{2}$, labelling the different broken symmetry states. This is the best choice at low temperatures, where we study the elementary excitations and topological defects. For studying the transition from low to high temperatures, when the magnetization goes to zero, it is better to consider $\vec{M}$ as a vector of varying length (a vector in $\mathcal{R}^{3}$ ). Finding the simplest description for your needs is often the key to the problem.

[8] Most magnets are crystals, which already have broken the rotational symmetry. For some "Heisenberg" mag- nets, the effects of the crystal on the magnetism is small. Magnets are really distinguished by the fact that they break time-reversal symmetry: if you reverse the arrow of time, the magnetization would change direction!

[9] We argue here that low frequency excitations come from spontaneously broken symmetries. They can also come from conserved quantities: since air cannot be created or destroyed, a long-wavelength density wave cannot relax quickly.

[10] In two dimensions, crystals provide another loophole in a well-known result, known as the Mermin-Wagner theorem. Hohenberg, Mermin, and Wagner, in a series of papers, proved in the 1960's that two-dimensional systems with a continuous symmetry cannot have a broken symmetry at finite temperature. At least, that's the English phrase everyone quotes when they discuss the theorem: they actually prove it for several particular systems, including superfluids, superconductors, magnets, and translational order in crystals. Indeed, crystals in two dimensions do not break the translational symmetry: at finite temperatures, the atoms wiggle enough so that the atoms don't sit in lock-step over infinite distances (their translational correlations decay slowly with distance). But the crystals do have a broken orientational symmetry: the crystal axes point in the same directions throughout space. (Mermin discusses this point in his paper on crystals.) The residual translational correlations (the local alignment into rows and columns of atoms) introduce long-range forces which force the crystalline axes to align, breaking the continuous rotational symmetry. Mermin, Wagner, and Hohenberg's methods apply very generally, but are not general enough to apply to this case (for good reason!)

[11] The next fashion, catastrophe theory, never became important for anything.

[12] This again is the mysterious lack of rotational Goldstone modes in crystals.

[13] The zeroth homotopy group classifies domain walls. The third homotopy group, applied to defects in threedimensional materials, classifies what the condensed matter people call textures and the particle people sometimes call skyrmions. The fourth homotopy group, applied to defects in space-time path integrals, classifies types of instantons.

[14] A loop is a continuous mapping from the circle into the order parameter space: $\theta \rightarrow u(\theta), 0 \leq \theta<2 \pi$. When we encircle the defect with a loop, we get a loop in order parameter space as shown in figure 4: $\theta \rightarrow \vec{x}(\theta)$ is the loop in real space, and $\theta \rightarrow u(\vec{x}(\theta))$ is the loop in order parameter space. Two loops are equivalent if there is a continuous one-parameter family of loops connecting one to the other: $u \equiv v$ if there exists $u_{t}(\theta)$ continuous both in $\theta$ and in $0 \leq t \leq 1$, with $u_{0} \equiv u$ and $u_{1} \equiv v$.

[15] That is, $u \otimes v(\theta) \equiv u(2 \theta)$ for $0 \leq \theta \leq \pi$, and $\equiv v(2 \theta)$ for $\pi \leq \theta \leq 2 \pi$. 